\title{
Half-Integer Filling-Factor States in Quantum Dots
}

\author{
A. Harju, ${ }^{1, *}$ H. Saarikoski, ${ }^{2,1}$ and E. Räsänen ${ }^{3,1}$ \\ ${ }^{1}$ Laboratory of Physics, Helsinki University of Technology, P.O. Box 1100, FI-02015 HUT, Finland \\ ${ }^{2}$ Kavli Institute of NanoScience, Delft University of Technology, 2628 CJ Delft, The Netherlands \\ ${ }^{3}$ Institut für Theoretische Physik, Freie Universität Berlin, Arnimallee 14, D-14195 Berlin, Germany
}

(Received 27 January 2006; published 29 March 2006)

\begin{abstract}
The emergence of half-integer filling-factor states, such as $\nu=5 / 2$ and $7 / 2$, is found in quantum dots by using numerical many-electron methods. These states have interesting similarities and differences with their counterstates found in the two-dimensional electron gas. The $\nu=1 / 2$ states in quantum dots are shown to have high overlaps with the composite fermion states. The lower overlap of the Pfaffian state indicates that electrons might not be paired in quantum dot geometry. The predicted $\nu=5 / 2$ state has a high spin polarization, which may have an impact on the spin transport through quantum dot devices.
\end{abstract}

PACS numbers: 73.21.La, 71.10.- w, 73.43.-f, 85.35.Be

The fractional quantum Hall state at filling factor $\nu=$ $5 / 2$ in the two-dimensional electron gas (2DEG) is widely thought to be a Moore-Read state of $p$-wave paired electrons with unusual topological properties [1]. The state has recently attracted much theoretical interest together with other half-integer filling-factor states, mainly due to proposals for using it in quantum computing [2]. The 2DEG $\nu=5 / 2$ state is expected to consist of two Landau levels (LLs), where the filled lowest LL (OLL) is spin compensated and the half-filled next LL (1LL) is a spin-polarized state described by a Pfaffian (PF) wave function [3]. This wave function explicitly describes the electron pairing which is analogous to that in the BCS theory of superconductivity. Because of the half filling of 1LL at $\nu=5 / 2$, the properties of the $\nu=5 / 2$ state are usually analyzed using the $\nu=1 / 2$ state with an interaction Hamiltonian corrected for the screening effects of the electrons in the filled OLL.

Unlike half-integer filling-factor states in the 2DEG, the corresponding states in quantum dots (QDs) have received almost no attention until now (see Ref. [4] for an exception). In this Letter we analyze the electronic structure of QDs in magnetic fields and find new states which can be regarded as finite-size counterparts of the half-integer filling-factor states in the 2DEG. Since the emergence of fractional quantum Hall states is always a result of electron-electron interactions, we use numerical methods which can take into account the complex correlation effects in the system. The results indicate analogies but also important differences between the states in the half-integer filling-factor range in the 2DEG and QDs. The mean-field method predicts that due to the presence of an external confining potential in QDs, the $\nu=5 / 2$ state appears as a mixture of $\nu=2$ and 3 . We analyze also the internal structure of the many-body state at $\nu=1 / 2$ in QDs and compare it to the $\mathrm{PF}$ wave function and the composite fermion (CF) model [5]. Unlike the CF model, which yields high overlaps with the exact state, the $\mathrm{PF}$ wave function is found to poorly describe the pure $\nu=1 / 2$ state and also the excited 1LL of the $\nu=5 / 2$ state in QDs. Therefore, we find no evidence of electron pairing near half-integer LL fillings in QDs. We finally discuss the impact of the $\nu=5 / 2$ state on the ground-state energetics and on the spin transport through the QD and find that signatures of this state can be found, e.g., in magnetization measurements or electron tunneling experiments.

We define our QD system by an effective-mass Hamiltonian for $N$ electrons:

$$
H=\sum_{i=1}^{N}\left[\frac{\left(\mathbf{p}_{i}+e \mathbf{A}\right)^{2}}{2 m^{*}}+V_{c}\left(r_{i}\right)+g^{*} \mu_{B} S_{z, i}\right]+\sum_{i<j} \frac{e^{2}}{4 \pi \epsilon r_{i j}},
$$

with material parameters for GaAs, i.e., $m^{*}=0.067$, $\epsilon / \epsilon_{0}=12.4$, and $g^{*}-0.44$. Unless stated otherwise, we apply a parabolic confining potential, $V_{c}(r)=m^{*} \omega_{0}^{2} r^{2} / 2$, with a strength $\hbar \omega_{0}=5.7 N^{-1 / 7} \mathrm{meV}$. The $N$ dependence in $\omega_{0}$ is introduced to keep the electron density in the dot approximately constant. We solve the associated Schrödinger equation using the mean-field spin-density functional theory (SDFT) and the exact diagonalization (ED) method [6]. We use the SDFT to calculate the electronic structure of large quantum dots which are beyond reach of exact many-body method. The internal structure of the half-filled LL is then analyzed in detail with the ED method which is, however, restricted to fairly low electron numbers.

We analyze the polarization and occupations of the LLs in large QDs using the SDFT. We focus here on the fillingfactor regime $\nu \geq 2$. One finds that the filling factor for the finite electron systems is position dependent [7]. Even at integer filling factors (where most physics can be understood on a single-particle level), the 2DEG and QDs have differences due to the overlapping of LLs in QDs in low magnetic fields. In QDs we identify the integer $\nu$ as states, where the increasing magnetic field has emptied one of the LLs completely of electrons. For example, $\nu=3$ corresponds to the case where magnetic field has emptied 2LL and the spin-compensated 1LL has approximately one- 
third of the electrons, the rest being at OLL. In the 2DEG, $1 \mathrm{LL}$ is spin split at $\nu=3$ and has one spin channel fully occupied.

Figure 1(a) shows the calculated spin polarization $S=$ $\left(N^{\uparrow}-N^{\downarrow}\right) / 2$ of the ground state of QDs with $N=12,30$, 48 , and 60 electrons, respectively. Our starting point is the $\nu=3$ state discussed above. When the magnetic field is increased, electrons move from 1LL to 0LL. This occurs mainly for spins antiparallel to the magnetic field. We find a peak in $S$ between $\nu=2$ and $\nu=3$ where all the spins antiparallel to the magnetic field have fallen to OLL leaving 1LL totally spin polarized with angular momentum states $l=-1,0,1, \ldots, n_{1 \mathrm{LL}}-2$. Since the spin polarization of 1LL agrees with the expected spin polarization of the Moore-Read state in the 2DEG, we identify this state as a finite-size counterpart of $\nu=5 / 2$. In addition, $\nu_{\mathrm{av}}=$ 2.54 (see Ref. [7]) for this state when $N=60$. The spin density of the $\nu=5 / 2$ state at $N=60$ shows that the spin polarization is concentrated at the core of the dot [Fig. 1(b)]. This result is in contrast with the structure of the 2DEG where the single-particle states on 1LL are uniformly occupied, whereas in QDs the occupation has a compact structure due to the external confinement. Thus, the $\nu=5 / 2$ state in QDs can be interpreted to be composed of two different filling-factor domains, i.e., $\nu=2$ at the edge and $\nu=3$ at the center. We find also an analogous state in lower magnetic fields $\nu>3$ where the $2 \mathrm{LL}$ is spin polarized. We identify this state as $\nu=7 / 2$. The KohnSham occupations of LLs for $\nu=2,5 / 2,3$, and $7 / 2$, respectively, show the mechanism of LL filling in QDs [Fig. 1(c)]. Because of the partial filling of 1LL, the $\nu=3$ state can also be interpreted as a combination of $\nu=2$ and $\nu=4$ states.

Increasing the magnetic field beyond $\nu=5 / 2$ forces the spin-polarized electrons in 1LL to fall one by one down to OLL, starting from the electrons with highest angular momenta. 1LL maintains the spin polarization, but the electrons in OLL tend to prefer spin-compensated configuration and, as a consequence, there is a partial relapse towards lower total spin polarization. However, near $\nu=20 \mathrm{LL}$ gets a small spin polarization in high electron numbers, e.g., $S=1$ for $N=48$ and $S=2$ for $N=60$ [see Fig. 1(a)]. This result is in accord with the results of Ciorga and co-workers who found the collapse of the spin singlet state in large QDs at $\nu=2$ (Ref. [9]).

The results at $\nu=5 / 2$ can be generalized to larger particle numbers by assuming a spin-compensated 0LL and polarized 1LL. The formula of $\nu_{0 L L}$ (see Ref. [7]) gives $S \approx N / 10$ for the spin polarization at $\nu=5 / 2$, which is in reasonable agreement with the calculated maximum spin polarization of the two largest dots in Fig. 1(a). As seen above in the case of $\nu=7 / 2$, we find also evidence of analogous polarization of the highest occupied LL in lower magnetic fields. This suggests that the results can be generalized to higher half-integer fillingfactor states $(\nu=9 / 2$, etc. $)$ in sufficiently large QDs. (a)

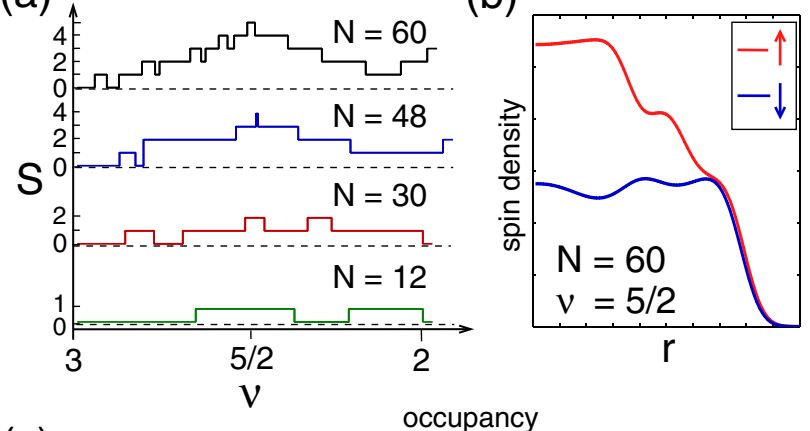

(c)

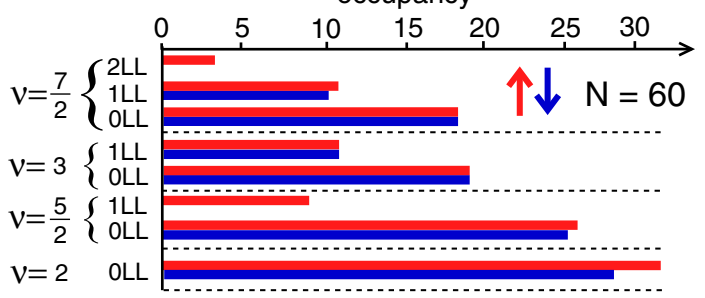

FIG. 1 (color online). (a) Ground-state spin of quantum dots with $N=12,30,48$, and 60 electrons, respectively. The magnetic fields are scaled linearly to filling-factor range $2 \leq \nu \leq 3$. (b) Spin densities of the $N=60$ quantum dot at $\nu=5 / 2$. The spin-polarized second Landau level gives rise to high spin polarization in the core region of the dot. (c) Occupation of the single-electron (Kohn-Sham) states on different Landau levels in the $N=60$ quantum dot at $2 \leq \nu \leq 7 / 2$.

The predicted spin polarization of the states between $2 \leq \nu \leq 3$ is interesting with a viewpoint on the spintronics in which electron spin degree is exploited to create novel device concepts and new functionality for electronic circuits. It is assumed that the alternation of states in the $2<\nu<3$ regime leads to the familiar checkerboard pattern of conductance peak heights in the electron tunneling through a QD device $[10,11]$. However, the details of the current patterns are not completely understood. The checkerboard behavior is usually explained assuming oscillations between the spin singlet $S=0$ and triplet $S=1$ states [12] which can be qualitatively modeled within the constant-interaction picture [13] with a phenomenological exchange term. Our SDFT calculations indicate that this model is sufficiently accurate in low electron numbers [see, e.g., data for $N=12$ in Fig. 1(a)], but there are significant deviations from it for $N \geq 20$. The constant-interaction model cannot take into account the correlation effects which give rise to the high spin polarization near the $\nu=$ $5 / 2$ state in large QDs. This might affect electron tunneling probability through the dot for high electron numbers and, as a consequence, leave a trace to the pattern of conductance peak heights. However, the high probability transmission in the experiments occurs mainly through the edge region which is not spin polarized [10]. The transport through the center of the dot gives rise to lower conductance peak heights, and evolution of the ground-state spin might leave a systematic pattern in the low probability transmission peak heights. 
We turn now to the analysis of the exact many-body wave function of the $\nu=1 / 2$ state. We use the ED method on OLL, and the results are compared to two models, the PF and the $\mathrm{CF}$ wave functions [5]. The PF wave function [1] is defined as

$$
\Psi_{\mathrm{PF}}=\operatorname{Pf}\left(\frac{1}{z_{i}-z_{j}}\right) \prod_{i<j}\left(z_{i}-z_{j}\right)^{2} \exp \left(-\frac{1}{2} \sum_{i} r_{i}^{2}\right),
$$

with angular momentum $L=N(N-1)-N / 2$. The value of the filling factor at $\nu<1$, i.e., beyond the maximumdensity-droplet (MDD) domain is widely approximated to be $\nu_{\mathrm{J}}=L_{\mathrm{MDD}} / L$. This definition is based on Jastrow states. For the PF wave function $\nu_{\mathrm{J}}$ is slightly higher than $1 / 2$. As the number of particles in the system increases, $\nu_{\mathrm{J}}$ for the $\mathrm{PF}$ approaches $1 / 2$ from above as $\nu_{\mathrm{J}}=$ $1 / 2+1 / 2 N+O(1 / N)^{2}$. The PF state is constructed for even $N$, and we are computationally limited to $N \leq 8$. The $\mathrm{CF}$ wave functions [5] used here are defined as

$$
\Psi_{\mathrm{CF}}=\exp \left(-\frac{1}{2} \sum_{i} r_{i}^{2}\right) \Psi_{0} \prod_{i<j}\left(z_{i}-z_{j}\right)^{2},
$$

where $\Psi_{0}$ is a determinant formed from the single-particle states $\psi_{n, l} \propto z^{n+l} \partial^{n}$, with $l$ the angular momentum and $n=$ $0,1,2, \ldots$, is the $L L$ index. The derivative is with respect to $z$, and the resulting many-body state has a total angular momentum $L=N(N-1)+\sum_{i=1}^{N} l_{i}$.

There are several possibilities for the single-particle occupations in $\Psi_{0}$ for the states near $\nu_{\mathrm{J}} \approx 1 / 2$. We consider two different CF wave functions: $\mathrm{CF} 1$ that have the same angular momentum as PF states, and CF2 that have $\nu_{\mathrm{J}}=1 / 2$, so that $L=N(N-1)=2 L_{\mathrm{MDD}}$. The first type has single-particle quantum numbers $(n, l)$ of the occupied states as $(0, i-1)$ and $(i,-i), i=1, \ldots, N / 2$, and in the second one the asymmetry in $l$ quantum numbers is recovered by moving a particle from $(N / 2,-N / 2)$ to $(1,0)$. The $\mathrm{CF} 2$ wave function is constructed also for odd $N$. The numerical projection to OLL (derivatives in $\Psi_{0}$ ) is doable up to $N=6$. From these wave functions, CF1 (and thus also $\mathrm{PF}$ ) has an angular momentum that corresponds to the ground state of $N=4$, and CF2 for $N=5$ and 6. In Table I we show the accuracy of the three wave functions discussed above. PF is clearly less accurate than $\mathrm{CF}$, which has very high overlaps. The reasonably high overlap (around 0.6) means, however, that PF cannot be an accurate excited state. Therefore we find no evidence of electron pairing in the $\nu=1 / 2$ state.

TABLE I. Overlaps of the trial wave functions with the exact one for different particle number $N$.

\begin{tabular}{cccc}
\hline \hline$N$ & PF & CF1 & CF2 \\
\hline 4 & 0.922124 & 0.999903 & 0.997978 \\
5 & $\ldots$ & $\ldots$ & 0.998921 \\
6 & 0.789996 & 0.993461 & 0.996096 \\
8 & 0.586370 & $\ldots$ & $\ldots$ \\
\hline \hline
\end{tabular}

Figure 2 shows the conditional wave functions [6], obtained by fixing $N-1$ electrons and moving the remaining electron, for the PF and corresponding ED state. One can see that the most probable positions are different in ED and PF, and the ED conditional density is more localized than the PF one.

In the 2DEG the electrons in the half-filled 1LL can be described by a $\nu=1 / 2$ state on OLL having a screened electron-electron interaction. This state is accurately modeled using a PF wave function [1,3]. The mean-field results above suggest that because of the different structure of the $\nu=5 / 2$ states in QDs and the 2DEG systems, the $\nu=5 / 2$ state is not related to $\nu=1 / 2$ in parabolically confined QDs. The 1LL in QDs is compact at $\nu=5 / 2$ and does not have the required angular momentum of the $\nu=1 / 2$ state. One might assume that realistic interelectron potentials, which are modified by screening from the electrodes and finite thickness of the sample, would induce electron pairing as observed in numerical studies of the $\nu=5 / 2$ state in the 2DEG. However, ED calculations with a screened electron-electron interaction indicate that overlaps of the $\mathrm{PF}$ with the exact state increases only marginally. Therefore we find no evidence of electron pairing in the analyzed half-integer filling-factor states in QDs confined by a parabolic external potential.

The confinement potential in actual QDs may vary considerably from the parabolic shape [14]. However, this fact seems not to affect notably the appearance of the $\nu=5 / 2$ state in QDs. Our SDFT calculations for a 60-electron QD defined by an infinite potential well of radius $150 \mathrm{~nm}$ yield the $\nu=5 / 2$ state similarly to the parabolic case. As the main difference, the state is not separated into two $\nu=2$ and $\nu=3$ domains in the infinite-well QD. Instead, electrons on 0LL are strongly localized near the edge, whereas the spin-up electrons on 1LL contribute in the midregion, leaving the electron density in the core relatively flat for both spin types. In the ground-state energetics the behavior between different QDs is similar, as we demonstrate in Fig. 3 showing the SDFT result of the magnetization, $M=$ $-\partial E_{\text {tot }} / \partial B$, for 60 -electron parabolic and infinite-well
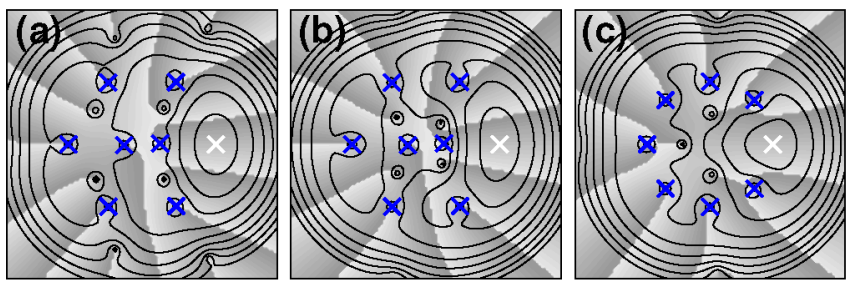

FIG. 2 (color online). Electron positions (crosses), conditional electron densities (contours), and phases (gray scale) of the $N=$ $8, L=52$ state from the (a) ED and (b), (c) PF models. We probe with the rightmost electron, and densities are on logarithmic scale. The phase changes from $\pi$ to $-\pi$ on the lines where the shadowing changes from the darkest gray to white. In (a) and (c) electrons are on the most probable positions, and in (b) we use ED coordinates for PF. 
(a)



(b)

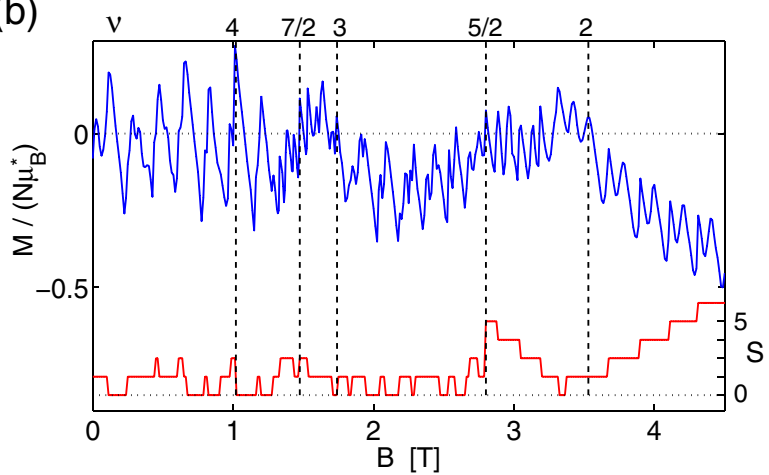

FIG. 3 (color online). Ground-state magnetization (upper curves) and total spins (lower curves) of 60-electron quantum dots in (a) parabolic and (b) infinite-well potentials. The finitesize counterparts of the integer and fractional quantum Hall states are marked in the panels (dashed lines).

QDs, respectively. The peaks in $M$ coincide with the integer filling factors and constitute the finite-size counterpart of the de Haas-van Alphen effect. In both cases, the $\nu=5 / 2$ state leaves cusps in the magnetization, and the data also show possible development of other half-integer filling-factor states such as $\nu=7 / 2$ in lower magnetic fields. The results are consistent with the few direct magnetization measurements of ensembles of large QDs which have shown a cusplike structure near $\nu=5 / 2$ (Ref. [15]). Visible signatures are also expected to be found in the chemical potentials of electron tunneling experiments.

To conclude, we predict emergence of states in quantum dots which can be regarded as finite-size counterparts of the half-integer filling-factor states in the 2D electron gas. The highest occupied Landau level of these states is found to be spin-polarized, and the formation of them leaves signatures in the ground-state energetics. The numerical results indicate that these states share many of the characteristics of their infinite size counterparts in the 2D electron gas, although we also find major differences. Because of the observed stability of these states, we postulate that the formation of them is a general property of finite fermion systems in external confinement. In parabolic confining potential the composite fermion picture is found to be a more accurate description of the $\nu=1 / 2$ state than the Pfaffian wave function, which suggests that electrons are not paired in this geometry. The electron pairing, which is predicted to occur in the two-dimensional electron gas, might be recovered in quantum dots in the limit of very large electron numbers and with a weak nonparabolic external confinement potential.

We thank S. Siljamäki for the $\mathrm{CF}$ wave function code, and J. Suorsa, G. E. W. Bauer, M. Schwarz, S. M. Reimann, and D. Grundler for fruitful discussions. This work was supported by the Academy of Finland through the Centre of Excellence Program (2000-2011) and by the NANOQUANTA NOE and the Finnish Academy of Science and Letters, Vilho, Yrjö and Kalle Väisälä Foundation (E. R.).

*Electronic address: ari.harju@hut.fi

[1] G. Moore and N. Read, Nucl. Phys. B360, 362 (1991).

[2] M. Freedman, C. Nayak, and K. Walker, cond-mat/ 0512066.

[3] E. H. Rezayi and F. D. M. Haldane, Phys. Rev. Lett. 84, 4685 (2000).

[4] A. Emperador, E. Lipparini, and F. Pederiva, Phys. Rev. B 72, 033306 (2005).

[5] J. K. Jain and R. K. Kamilla, Int. J. Mod. Phys. B 11, 2621 (1997).

[6] H. Saarikoski, A. Harju, M. J. Puska, and R. M. Nieminen, Phys. Rev. Lett. 93, 116802 (2004).

[7] One possibility to define $\nu$ of a particular state in a QD is from the number of electrons on 0LL $\left(N_{0 \mathrm{LL}}\right)$ as $\nu_{0 \mathrm{LL}}=$ $2 N / N_{\text {OLL }}$. A second option is by Kinaret et al. [8], who defined the average filling factor as $\nu_{\mathrm{av}}=N^{2} / 2(N+L)$, where $L$ is the total angular momentum. These two definitions differ especially in the low magnetic field range.

[8] J.M. Kinaret, Y. Meir, N.S. Wingreen, P. A. Lee, and X. G. Wen, Phys. Rev. B 46, 4681 (1992).

[9] M. Ciorga, A. Wensauer, M. Pioro-Ladriere, M. Korkusinski, J. Kyriakidis, A. S. Sachrajda, and P. Hawrylak, Phys. Rev. Lett. 88, 256804 (2002).

[10] M. Keller, U. Wilhelm, J. Schmid, J. Weis, K. v. Klitzing, and K. Eberl, Phys. Rev. B 64, 033302 (2001).

[11] K. Hitachi, M. Yamamoto, and S. Tarucha (unpublished).

[12] S. Tarucha, D. G. Austing, Y. Tokura, W. G. van der Wiel, and L. P. Kouwenhoven, Phys. Rev. Lett. 84, 2485 (2000).

[13] M. Stopa, W. G. van der Wiel, S. De Franceschi, S. Tarucha, and L. P. Kouwenhoven, Phys. Rev. Lett. 91, 046601 (2003).

[14] N. A. Bruce and P. A. Maksym, Phys. Rev. B 61, 4718 (2000).

[15] M. P. Schwarz, D. Grundler, Ch. Heyn, D. Heitmann, D. Reuter, and A. Wieck, Phys. Rev. B 68, 245315 (2003). 